\title{
Ultrasound diagnosis of nephrocalcinosis in preterm infants
}

\author{
N WOOLFIELD,${ }^{*} \mathrm{R}$ HASLAM,$\dagger \mathrm{G}$ LE QUESNE, $\ddagger \mathrm{H}$ M CHAMBERS $\uparrow \mathrm{R}$ HOGG, ${ }^{*}$ \\ AND K JUREIDINI*
}

Departments of ${ }^{*}$ Nephrology and $\ddagger$ Diagnostic Ultrasound, Adelaide Children’s Hospital, and $\dagger$ Neonatal
Intensive Care Unit, and $\lceil$ Department of Pathology, Queen Victoria Hospital, Adelaide, South Australia.

SUMmaRY The incidence of nephrocalcinosis in very low birthweight (less than $1500 \mathrm{~g}$ ) premature infants was assessed by ultrasound scan and analysis of urine. Three of 36 infants had nephrocalcinosis. All had been receiving long term frusemide for bronchopulmonary dysplasia with simultaneous fluid restriction. Urinary investigations showed no consistent findings in babies with nephrocalcinosis.

The survival of very low birthweight premature infants has improved in the last 10 years as advances in the understanding of diseases and their treatment have been made. Multiple drug treatment is often required, and metabolic disturbances are common.

As more infants survive, a larger number who initially required assisted ventilation and increased oxygen concentrations develop chronic lung disease which requires long term oxygen supplements. Frusemide is sometimes used for several months while the babies are dependent on oxygen. The oral dose ranges from $2-8 \mathrm{mg} / \mathrm{kg} /$ day. Side effects include hyponatraemia, hypokalaemia, hypochloraemic metabolic alkalosis, and hypercalciuria. ${ }^{1}$ Urinary calcium excretion is increased 10 -fold $^{2}$ and this is thought to be the major factor in the development of nephrocalcinosis and nephrolithiasis. ${ }^{3}$ The total dose may also be important. Osteopenia, from which premature infants are at risk, is also exacerbated.

Early studies used abdominal radiographs to detect nephrocalcinosis ${ }^{2}$; more recent reports have shown that real time ultrasound is considerably more sensitive. ${ }^{3}$ Ultrasound pictures show nephrocalcinosis as areas of increased echo production in the medullary pyramids. They may also detect associated renal calculi.

\section{Patients and methods}

Infants admitted to the neonatal unit at the Queen Victoria Hospital with birth weights of $\leqslant 1500 \mathrm{~g}$ born between 1 Septémber 1983 and 30 July 1984 were included in the study.

At 12 months of age all infants had ultrasound scans of the urinary tract with a real time sector scanner using a $7.5 \mathrm{MHz}$ or $5 \mathrm{MHz}$ transducer. Parasagittal and coronal views of the kidneys were routinely taken, and the coronal views usually provided the best definition of the anatomy of the kidney.

A spot sample of urine was centrifuged and examined by phase contrast microscopy. An aliquot was simultaneously frozen for batch analysis of osmolality, and calcium, creatinine, and $\beta-2-$ microglobulin concentrations using the Phadebas radioimmunoassay (Pharmacia Diagnostics).

\section{Results}

Twenty eight of 64 infants were excluded, 20 because of geographical isolation, and eight because the parents did not give consent.

Two of four infants who weighed less than $1000 \mathrm{~g}$ at birth and one of 32 who weighed between 1000 and $1501 \mathrm{~g}$ at birth had nephrocalcinosis. The dose of frusemide that had been given to the three with nephrocalcinosis varied from 2 to $8 \mathrm{mg} / \mathrm{kg} / \mathrm{day}$. The total doses in the individual infants ranged from $220-630 \mathrm{mg}$. All three were on fluid regimens of less than $160 \mathrm{ml} / \mathrm{kg} / \mathrm{day}$ for at least $80 \%$ of the time that they were receiving frusemide. None had received chlorothiazide. Of the 33 infants who did not have nephrocalcinosis, the total frusemide dose in individual infants ranged from $0-305 \mathrm{mg}$; only two received more than $100 \mathrm{mg}$. Both received more than $170 \mathrm{ml} / \mathrm{kg} /$ day of fluid.

In the intensive care unit management (including nutrition and vitamin supplementation) is standardised. There was no difference between the infants with and without nephrocalcinosis in the amount of vitamin D supplementation, or the type of milk mixture they received, or the incidence of osteopenia of prematurity or prolonged acidosis and hypercalcaemia, or the administration of other drugs.

Urinary $\beta-2$-microglobulin was within the normal range $(0-375 \mathrm{ng} / \mathrm{ml})$ in all infants. Urinary calcium: creatine ratio $(\mathrm{mmol}: \mathrm{mmol})$ ranged from 0.033 3.68 (normal $<0.6)$ and this did not correlate in any way with nephrocalcinosis. Microscopy of the urine did not show any haematuria or other abnormalities 
in any infant, and osmolality and $\mathrm{pH}$ varied, but there were no associations between any measurement and nephrocalcinosis.

In two of the three infants the nephrocalcinosis resolved. In one it disappeared after 16 months when the frusemide was stopped. In the second it disappeared after five months' treatment with chlorothiazide. The third died of unrelated causes and necropsy was not performed.

In addition to the three infants with nephrocalcinosis, five were found to have structural abnormalities of the urinary tract on ultrasound scans.

\section{Discussion}

Ultrasound scanning has proved to be a useful and effective means of diagnosing nephrocalcinosis. Correlation of results of ultrasound scanning before death and histology at necropsy has confirmed the association between increased echogenicity of medullary pyramids and nephrocalcinosis. ${ }^{2}$ (figs 1 and 2). The greater sensitivity of modern ultrasound equipment in diagnosing nephrocalcinosis suggests that early reports may have underestimated its incidence. $^{2}$ Nephrocalcinosis is associated with hypercalcaemia and hypercalciuria, hypervitaminosis $\mathrm{D}$, primary hyperoxaluria, distal renal tubular acidosis, and Bartter's syndrome. It also occurs in premature infants on long term frusemide treatment, and in children taking vitamin D and phosphate for X-linked hypophosphaturic rickets. It has recently been observed in association with a hypoprostaglandinuric tubular syndrome with hypercalciuria and hypokalaemia in preterm infants. $^{4}$

The earliest calcium deposits appear as an echogenic rim at the periphery of the medullary pyramids with a preserved echolucent centre. As the disease progresses the entire medullary pyramid becomes echogenic. ${ }^{5}$

Nephrocalcinosis should be differentiated from other causes of increased echogenicity of medullary pyramids. During the neonatal period oliguria associated with medullary hyperechogenicity may indicate the transient acute tubular disease that is due to deposition of Tamm-Horsfall glyco protein. Several earlier case reports of nephrocalcinosis may have been describing this disease, ${ }^{6}$ as follow up ultrasound scans were not always done. Differentiation between the two diseases is possible if the ultrasound examination is repeated after two weeks. In transient echogenicity the echogenic regions resolve over days, whereas nephrocalcinosis takes months. Recessive polycystic renal disease may produce a pattern of echogenic pyramids in children, but in infants the kidneys are usually

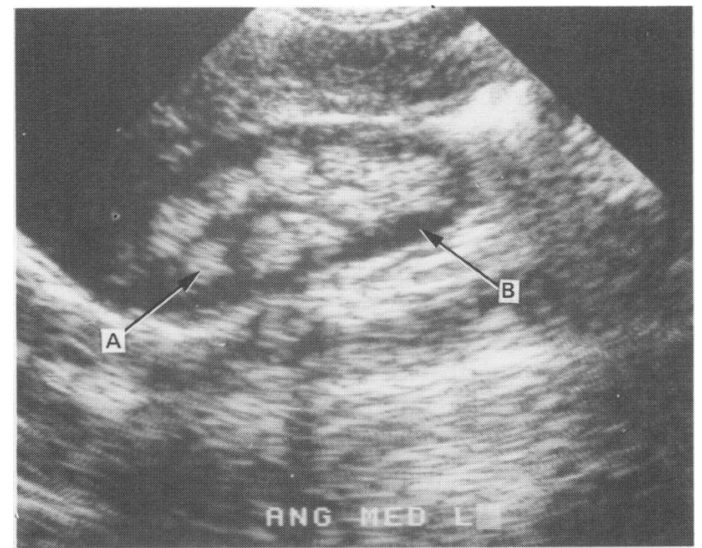

Fig 1 Renal ultrasound picture showing medullary nephrocalcinosis. Medullary pyramids $(A)$ are densely and abnormally echogenic; renal cortex $(B)$ is normal.

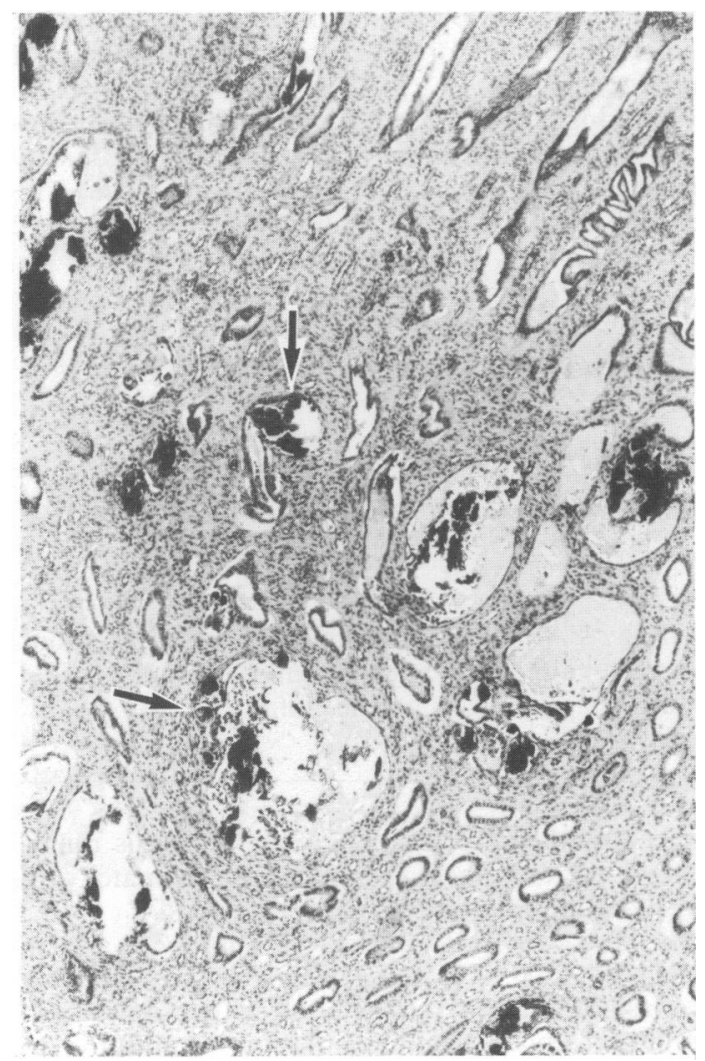

Fig 2 Photomicrograph of renal pyramid showing extensive nephrocalcinosis. Dense interstitial and intratubular calcium deposits (arrows) show staining and polarisation characteristics of mixed calcium oxalate and phosphate. (Haemotoxylin and eosin.) 
enlarged and diffusely echogenic. Juvenile familial nephronophthisis may also show increased echo formation, particularly around the periphery of the medullary pyramids and associated with microcyst formation. Some larger cysts may also be visible.

Unlike Seyberth et al we found no abnormalities in tests of tubular function. ${ }^{4}$ Microscopic haematuria has been seen in infants with nephrocalcinosis who are given frusemide, but it does not seem to be associated specifically with nephrocalcinosis.

The combined use of chlorothiazide with frusemide has been advocated as a means of preventing hypercalciuria. It also aids the dissolution of nephrocalcinosis and any stones that may be present. ${ }^{2}$ We have shown that nephrocalcinosis can disappear without using chlorothiazide over a period of 16 months after stopping the frusemide. The most valuable use of chlorothiazide may be in those infants who have developed kidney stones ${ }^{2}$ and in those with osteopenia of prematurity. The volume of fluid tolerated by the infant receiving frusemide seems to be important in the development of nephrocalcinosis.

The long term effects of medullary nephrocalcinosis are unknown, but may be related to the density of calcium present and the underlying cause. The absence of tubular abnormalities in our group at 12 months suggests that they did not have any ill effects.

We thank Miss Gail Simons and Mrs Meg Hogg for typing the manuscript.

\section{References}

${ }^{1}$ Ross B, Pollak A, Oh W. The pharmacologic effects of furosemide therapy in the low birth weight infant. $J$ Pediatr 1978;92:149-52.

2 Hufnagle KG, Khan SN, Penn D, Cacciarelli A, Williams P. Renal calcifications: a complication of long term furosemide therapy in premature infants. Pediatrics 1982;70:360-3.

${ }^{3}$ Pearse DM, Kaude JV, Williams JL, Bush D, Wright PG. Sonographic diagnosis of furosemide induced nephrocalcinosis in newborn infants. J Ultrasound Med 1984;3:553-6.

${ }^{4}$ Seyberth HW, Rascher W, Schweer H, Kuhl PG, Mehls SO, Scharer K. Congenital hypokalaemia with hypercalciuria in preterm infants: a hyperprostaglandinuric tubular syndrome different from Bartter syndrome. J Pediatr 1985;107:694-701.

5 Patriquin $\mathrm{H}$, Robitaille $\mathrm{P}$. Renal calcium deposition in children: sonographic evaluation of the Anderson-Carr progression. $A J R$ 1986;146:1253-6.

6 Filiatraut D, Perraeult G. Transient acute tubular disease in a newborn and a young infant. J Ultrasound Med 1985;11:326-7.

Correspondence to Dr KF Jureidini, Department of Nephrology, Adelaide Children's Hospital, North Adelaide, South Australia 5006.

Received 7 July 1987

\title{
Ranitidine in the newborn
}

\author{
M ROSENTHAL AND P W MILLER
}

North West Regional Perinatal Centre, St Mary's Hospital, Manchester

SUMmarY An infusion of ranitidine $0.2 \mathrm{mg} / \mathrm{kg} /$ hour abruptly halted a life threatening gastrointestinal haemorrhage in an anuric infant of 30 weeks' gestation.

We report the successful use of intravenous ranitidine in an extremely sick preterm neonate with severe acute gastrointestinal blood loss.

She was born at 30 weeks' gestation with a birth weight of $1700 \mathrm{~g}$ to a healthy primigravida. She required brief initial resuscitation with endotracheal intubation and assisted ventilation in addition to naloxone. Vitamin $\mathrm{K}$ was given intramuscularly. The infant developed respiratory distress and collapsed when 5 hours old with a generalised convulsion that required further intubation and assisted ventilation. She remained hypoxic despite vigorous ventilation, with the fractional inspired oxygen concentration being raised to $95 \%$, and persistent fetal circulation was diagnosed. A bolus dose of tolazoline $2 \mathrm{mg} / \mathrm{kg}$ was given followed by an infusion at $2 \mathrm{mg} / \mathrm{kg} / \mathrm{hour}$, which produced a good initial response. She was transferred to the regional neonatal intensive care unit at the age of 12 hours.

Chest $x$ ray pictures showed severe hyaline membrane disease and the baby continued to shunt with a $\mathrm{PaO}_{2}$ of only $5.33 \mathrm{kPa}$ despite ventilation with $95 \%$ oxygen and infusions of tolazoline and dopamine $(5 \mu \mathrm{g} / \mathrm{kg} /$ minute $)$. At 40 hours of age she developed a loud murmur consistent with a persistent ductus arteriosus; this responded within nine hours to indomethacin $0.2 \mathrm{mg} / \mathrm{kg}$ and three doses were given 12 hourly intravenously.

On the third day she became anuric; renal ultrasound scan suggested acute tubular necrosis, and she required peritoneal dialysis for 36 hours for 